\title{
A new strongly convergent algorithm to solve pseudo- monotone equilibrium problems in a real Hilbert space
}

\author{
Kanikar Muangchoo
}

Faculty of Science and Technology, Rajamangala University of Technology Phra Nakhon (RMUTP), 1381 Pracharat 1 Road, Wongsawang, Bang Sue, Bangkok 10800, Thailand.

\begin{abstract}
The purpose of this research is to formulate a new algorithm by combining a viscosity-type method with the extragradient algorithm and explicit step size rule to figure out the equilibrium problems involving pseudo-monotone and Lipschitz-type continuous bi-function in a real Hilbert space. A strong convergence theorem is well-established by the use of certain mild conditions on the bi-function, as well as some conditions on the iterative control parameters. The designed algorithm uses a non-monotonic step size rule based on the local bi-function information. Applications of the main results are also presented to solve variational inequalities and fixed-point problems. The computational behaviour of the designed algorithm on a test problem is performed related to other existing algorithms.
\end{abstract}

Keywords: Equilibrium problem, strong convergence, viscosity method, Lipschitz-type conditions, fixed point problems, variational inequality problem.

2020 MSC: 47J25, 47H09, 47H06, 47J05.

(C)2022 All rights reserved.

\section{Introduction}

Assume that $\mathcal{K}$ is a closed and convex subset of a real Hilbert space $\mathcal{E}$ with the inner product and the induced norm are denoted by $\langle\cdot, \cdot\rangle$ and $\|\cdot\|$, respectively. Let $f: \mathcal{E} \times \mathcal{E} \rightarrow \mathbb{R}$ be a bi-function and satisfying $f(y, y)=0$ for all $y \in \mathcal{K}$. An equilibrium problem (EP) [5] for a bi-function $f$ on $\mathcal{K}$ is defined by

$$
\text { Find } \wp^{*} \in \mathcal{K} \text { such that } f\left(\wp^{*}, y\right) \geqslant 0, \forall y \in \mathcal{K} \text {. }
$$

Moreover, $S_{E P}$ represented a solution set of a problem (EP) over the set $\mathcal{K}$ and $\wp^{*}$ is any random element of $S_{\text {EP. }}$. A metric projection $\mathrm{P}_{\mathcal{K}}(u)$ of $u \in \mathcal{E}$ onto a closed and convex subset $\mathcal{K}$ of $\mathcal{E}$ is defined by

$$
\mathrm{P}_{\mathcal{K}}(\mathrm{u})=\underset{\mathrm{y} \in \mathcal{K}}{\arg \min }\|\mathrm{y}-\mathrm{u}\| .
$$

The problem (EP) is a general mathematical problem in the sense that it brings together many numerical problems, i.e., the fixed point problems, the vector and scalar minimization problems, the problems of

Email address: kanikar.m@rmutp.ac.th (Kanikar Muangchoo)

doi: $10.22436 /$ jmcs.024.04.03

Received: 2020-10-25 Revised: 2021-01-29 Accepted: 2021-02-23 
variational inequalities (VIP), complementarity problems, saddle point problems, the Nash equilibrium problems in non-cooperative games and the inverse optimization problems $[5,17]$. The problem $(\mathrm{EP})$ is also known as the well-established Ky Fan inequality due to his initial contribution [8]. Due to the importance of the problem (EP) and its applications in both pure and applied sciences, many researchers have investigated it broadly in recent years $[1,4,18,22,23,26,33]$ and others in $[10,19-21,24,25,27,28]$.

An important method is introduced by Tran et al. in [31] and established an iterative sequence $\left\{u_{n}\right\}$ in the following way:

$$
\left\{\begin{array}{l}
u_{0} \in \mathcal{K}, \\
y_{n}=\underset{y \in \mathcal{K}}{\arg \min }\left\{\chi f\left(u_{n}, y\right)+\frac{1}{2}\left\|u_{n}-y\right\|^{2}\right\}, \\
u_{n+1}=\underset{y \in \mathcal{K}}{\arg \min }\left\{\chi f\left(y_{n}, y\right)+\frac{1}{2}\left\|u_{n}-y\right\|^{2}\right\},
\end{array}\right.
$$

where $0<\chi<\min \left\{\frac{1}{2 c_{1}}, \frac{1}{2 c_{2}}\right\}$. It is important to note that some established methods are using constant step sizes based on bi-functional Lipschitz-type coefficients as well as weak provides convergence $[9,11$, 13, 31].

So, a reasonable question arises:

"Is it possible to introduce a new strongly convergent extragradient type method with non-monotone step size rule to solve the equilibrium problem"?

In this paper, we present a positive answer to this question, i.e., the gradient method still provides a strong convergence sequence by using a non-monotonic step size rule for solving equilibrium problems accompanied with pseudo-monotone functions. Inspired by the works of Censor et al. [7] and Moudafi [16] we introduce a new viscosity-type gradient method to figure out the problem (EP) in the context of infinite-dimensional real Hilbert spaces.

In particular, the key contributions in this paper are listed below:

$\triangleright$ We introduce an explicit subgradient viscosity method with a non-monotone step size rule to solve the equilibrium problem in a real Hilbert space. In the proposed iterative scheme, we do consider that the bi-function of the (EP) problem is a pseudo-monotone.

$\triangleright$ We establish a strong convergence result under mild conditions and suitable iterative control parameters frameworks.

$\triangleright$ Applications of our main results are studied to solve particular classes of equilibrium problems in real Hilbert spaces.

$\triangleright$ We provide numerical descriptions of our method for confirming the theoretical findings and comparing the results in [Algorithm 3.2 in [11]] and [Algorithm 3.1 in [12]]. Our numerical results indicate that the proposed method is effective compared to the existing ones.

The remainder of this article has been organized as follows: Section 2 includes some preliminary and basic results. Section 3 contains the proposed algorithm and the corresponding strong convergence result. Section 4 contains applications of our main results. Section 5 involves the numerical discussion of the proposed method compared to existing ones.

\section{Preliminaries}

In this article, the equilibrium problem is studied based on the following conditions. A bi-function $f: \mathcal{E} \times \mathcal{E} \rightarrow \mathbb{R}$ is said to be $[3,5])$ :

(Ф1) pseudo-monotone on $\mathcal{K}$ if

$$
f\left(y_{1}, y_{2}\right) \geqslant 0 \Longrightarrow f\left(y_{2}, y_{1}\right) \leqslant 0, \forall y_{1}, y_{2} \in \mathcal{K}
$$


(Ф2) Lipschitz-type continuous [15] on $\mathcal{K}$ if there exist two constants $c_{1}, c_{2}>0$ such that

$$
f\left(y_{1}, y_{3}\right) \leqslant f\left(y_{1}, y_{2}\right)+f\left(y_{2}, y_{3}\right)+c_{1}\left\|y_{1}-y_{2}\right\|^{2}+c_{2}\left\|y_{2}-y_{3}\right\|^{2}, \forall y_{1}, y_{2}, y_{3} \in \mathcal{K} .
$$

(Ф3) $\limsup _{n \rightarrow \infty} f\left(u_{n}, y\right) \leqslant f\left(p^{*}, y\right)$ for all $y \in \mathcal{K}$ and $\left\{u_{n}\right\} \subset \mathcal{K}$ satisfies $u_{n} \rightarrow p^{*}$.

(Ф4) $f(u, \cdot)$ is sub-differentiable and convex on $\mathcal{E}$ for every each $u \in \mathcal{E}$.

A normal cone of $\mathcal{K}$ at $u \in \mathcal{K}$ is defined by

$$
\mathrm{N}_{\mathcal{K}}(\mathrm{u})=\{w \in \mathcal{E}:\langle w, y-u\rangle \leqslant 0, \forall y \in \mathcal{K}\} .
$$

Let $\varphi: \mathcal{K} \rightarrow \mathbb{R}$ is convex function. The subdifferential of $\varphi$ at $u \in \mathcal{K}$ is defined by

$$
\partial \varphi(\mathfrak{u})=\{w \in \mathcal{E}: \varphi(\mathfrak{y})-\varphi(\mathfrak{u}) \geqslant\langle w, y-u), \forall y \in \mathcal{K}\} .
$$

Lemma 2.1 ([2]). Assume that $\mathrm{P}_{\mathcal{K}}: \mathcal{E} \rightarrow \mathcal{K}$ is a metric projection such that

$$
\left\|y_{1}-P_{\mathcal{K}}\left(y_{2}\right)\right\|^{2}+\left\|P_{\mathcal{K}}\left(y_{2}\right)-y_{2}\right\|^{2} \leqslant\left\|y_{1}-y_{2}\right\|^{2}, y_{1} \in \mathcal{K}, y_{2} \in \mathcal{E} .
$$

(ii) $\mathrm{y}_{3}=\mathrm{P}_{\mathcal{K}}\left(\mathrm{y}_{1}\right)$ if and only if

$$
\left\langle y_{1}-y_{3}, y_{2}-y_{3}\right\rangle \leqslant 0, \forall y_{2} \in \mathcal{K}
$$

$$
\left\|y_{1}-P_{\mathcal{K}}\left(y_{1}\right)\right\| \leqslant\left\|y_{1}-y_{2}\right\|, y_{2} \in \mathcal{K}, y_{1} \in \mathcal{E} \text {. }
$$

Lemma 2.2 ([32]). Assume that $\left\{p_{n}\right\} \subset(0,+\infty)$ is a sequence satisfying the following condition

$$
p_{n+1} \leqslant\left(1-\gamma_{n}\right) p_{n}+\gamma_{n} \delta_{n}, \forall n \in \mathbb{N},
$$

where $\left\{\gamma_{n}\right\} \subset(0,1)$ and $\left\{\delta_{n}\right\} \subset \mathbb{R}$ such that

$$
\lim _{n \rightarrow \infty} \gamma_{n}=0, \sum_{n=1}^{\infty} \gamma_{n}=+\infty \text { and } \limsup _{n \rightarrow \infty} \delta_{n} \leqslant 0 .
$$

Then, $\lim _{n \rightarrow \infty} p_{n}=0$.

Lemma 2.3 ([14]). Assume that a sequence $\left\{p_{n}\right\} \subset \mathbb{R}$ and there exists a subsequence $\left\{n_{i}\right\}$ of $\{n\}$ such that $p_{n_{i}}<$ $p_{n_{i+1}}$, for all $i \in \mathbb{N}$. Then, there is a non decreasing sequence $m_{k} \subset \mathbb{N}$ such that $m_{k} \rightarrow \infty$ as $k \rightarrow \infty$, and the following conditions are fulfilled by all (sufficiently large) numbers $k \in \mathbb{N}$ :

$$
p_{m_{k}} \leqslant p_{m_{k+1}} \text { and } p_{k} \leqslant p_{m_{k+1}} .
$$

In fact, $m_{k}=\max \left\{j \leqslant k: p_{j} \leqslant v_{j+1}\right\}$.

Lemma 2.4 ([2]). For each $\mathrm{y}_{1}, \mathrm{y}_{2} \in \mathcal{E}$ and $\delta \in \mathbb{R}$, then the following relationships hold.

$$
\left\|\delta y_{1}+(1-\delta) y_{2}\right\|^{2}=\delta\left\|y_{1}\right\|^{2}+(1-\delta)\left\|y_{2}\right\|^{2}-\delta(1-\delta)\left\|y_{1}-y_{2}\right\|^{2}
$$

$$
\left\|y_{1}+y_{2}\right\|^{2} \leqslant\left\|y_{1}\right\|^{2}+2\left\langle y_{2}, y_{1}+y_{2}\right\rangle .
$$

Lemma 2.5 ([29]). Let $\varphi: \mathcal{K} \rightarrow \mathbb{R}$ be a proper convex, lower semi-continuous and sub-differentiable function on $\mathcal{K}$. Then, $u \in \mathcal{K}$ minimize the function $\varphi$ on $\mathcal{K}$ iff

$$
0 \in \partial \varphi(u)+N_{\mathcal{K}}(u)
$$

where $\partial \varphi(\mathrm{u})$ stands for the sub-differential of $\varphi$ at $\mathrm{u} \in \mathcal{K}$ and $\mathrm{N}_{\mathcal{K}}(\mathrm{u})$ a normal cone of $\mathcal{K}$ at $\mathrm{u}$. 


\section{Main Results}

In this section, we present an iterative scheme for solving pseudo-monotone equilibrium problems that is based on Tran et al. in [31] and viscosity scheme [16]. It is important to note that the proposed method has a simple structure for achieving strong convergence. Suppose that $\mathrm{g}: \mathcal{E} \rightarrow \mathcal{E}$ be a strict contraction function with constant $\xi \in[0,1)$. The main algorithm has been presented as follows:

Algorithm 1 (A viscosity method for pseudo-monotone equilibrium problems)

Step 0: Choose $u_{0} \in \mathcal{K}, 0<\sigma<\min \left\{1, \frac{1}{2 c_{1}}, \frac{1}{2 c_{2}}\right\}, \mu \in(0, \sigma), \chi_{0}>0$ and a sequence $\alpha_{n} \subset(0,1)$ meet the following conditions:

$$
\lim _{n \rightarrow \infty} \alpha_{n}=0 \text { and } \sum_{n}^{+\infty} \alpha_{n}=+\infty \text {. }
$$

Step 1: Compute

$$
y_{n}=\underset{y \in \mathcal{K}}{\arg \min }\left\{\chi_{n} f\left(u_{n}, y\right)+\frac{1}{2}\left\|u_{n}-y\right\|^{2}\right\}
$$

If $u_{n}=y_{n}$, then stop the sequence. Otherwise, go to Step 2.

Step 2: Construct a half-space

$$
\mathcal{E}_{n}=\left\{z \in \mathcal{E}:\left\langle u_{n}-x_{n} \omega_{n}-y_{n}, z-y_{n}\right\rangle \leqslant 0\right\},
$$

where $\omega_{n} \in \partial_{2} f\left(u_{n}, y_{n}\right)$ satisfying $u_{n}-\chi_{n} \omega_{n}-y_{n} \in N_{\mathcal{K}}\left(y_{n}\right)$. Compute

$$
z_{n}=\underset{y \in \mathcal{E}_{n}}{\arg \min }\left\{\mu \chi_{n} f\left(y_{n}, y\right)+\frac{1}{2}\left\|u_{n}-y\right\|^{2}\right\}
$$

Step 3: Compute

$$
u_{n+1}=\alpha_{n} g\left(u_{n}\right)+\left(1-\alpha_{n}\right) z_{n} .
$$

Step 4: Compute

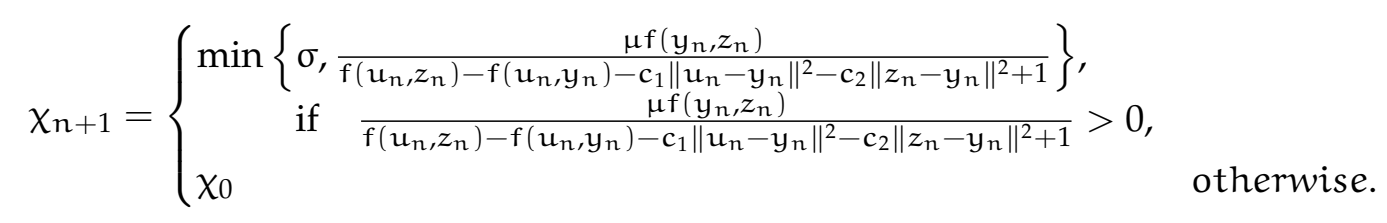

Set $n:=n+1$ and go back to Step 1 .

Lemma 3.1. Suppose that the conditions $(\Phi 1)-(\Phi 4)$ are satisfied. Then, the sequence $\left\{u_{n}\right\}$ generated by Algorithm 1 is a bounded sequence.

Proof. By the use of definition of $z_{\mathfrak{n}}$, we obtain

$$
0 \in \partial_{2}\left\{\mu \chi_{n} f\left(y_{n}, y\right)+\frac{1}{2}\left\|u_{n}-y\right\|^{2}\right\}\left(z_{n}\right)+N_{\mathcal{K}}\left(z_{n}\right) .
$$

Therefore, there exists a $\omega_{n} \in \partial_{2} f\left(y_{n}, z_{n}\right)$ and $\bar{\omega}_{n} \in N_{\mathcal{K}}\left(z_{n}\right)$ such that

$$
\mu \chi_{n} \omega_{n}+z_{n}-u_{n}+\bar{\omega}_{n}=0 .
$$

This relationship implies that

$$
\left\langle u_{n}-z_{n}, y-z_{n}\right\rangle=\mu \chi_{n}\left\langle\omega_{n}, y-z_{n}\right\rangle+\left\langle\bar{\omega}_{n}, y-z_{n}\right\rangle, \forall y \in \mathcal{K} .
$$


Due to $\bar{\omega}_{n} \in N_{\mathcal{K}}\left(z_{n}\right)$ imply that $\left\langle\bar{\omega}_{n}, y-z_{n}\right\rangle \leqslant 0$, for every $y \in \mathcal{K}$. Thus, we have

$$
\mu \chi_{n}\left\langle\omega_{n}, y-z_{n}\right\rangle \geqslant\left\langle u_{n}-z_{n}, y-z_{n}\right\rangle, \forall y \in \mathcal{K} .
$$

By given $\omega_{n} \in \partial_{2} f\left(y_{n}, z_{n}\right)$, we get

$$
f\left(y_{n}, y\right)-f\left(y_{n}, z_{n}\right) \geqslant\left\langle\omega_{n}, y-z_{n}\right\rangle, \forall y \in \mathcal{K}
$$

From (3.2) and (3.3), we get

$$
\mu \chi_{n} f\left(y_{n}, y\right)-\mu \chi_{n} f\left(y_{n}, z_{n}\right) \geqslant\left\langle u_{n}-z_{n}, y-z_{n}\right\rangle, \forall y \in \mathcal{K}
$$

In similar way $y_{n}$ gives that

$$
x_{n}\left\{f\left(u_{n}, y\right)-f\left(u_{n}, y_{n}\right)\right\} \geqslant\left\langle u_{n}-y_{n}, y-y_{n}\right\rangle, \forall y \in \mathcal{K}
$$

By the use of $y=z_{n}$ into (3.5), we get

$$
x_{n}\left\{f\left(u_{n}, z_{n}\right)-f\left(u_{n}, y_{n}\right)\right\} \geqslant\left\langle u_{n}-y_{n}, z_{n}-y_{n}\right\rangle .
$$

By the use of $y=\wp^{*}$ into (3.4), we get

$$
\mu \chi_{n} f\left(y_{n}, \wp^{*}\right)-\mu \chi_{n} f\left(y_{n}, z_{n}\right) \geqslant\left\langle u_{n}-z_{n}, \wp^{*}-z_{n}\right\rangle
$$

Since $\wp^{*} \in S_{E P}$, this implies that $f\left(\wp^{*}, y_{n}\right) \geqslant 0$ and pseudo-monotonicity of a bi-function $f$ provides $f\left(y_{n}, \wp^{*}\right) \leqslant 0$. Thus, (3.6) implies that

$$
\left\langle u_{n}-z_{n}, z_{n}-\wp^{*}\right\rangle \geqslant \mu \chi_{n} f\left(y_{n}, z_{n}\right) .
$$

From (3.1), we have

$$
\mu f\left(y_{n}, z_{n}\right) \geqslant x_{n+1}\left[f\left(u_{n}, z_{n}\right)-f\left(u_{n}, y_{n}\right)-c_{1}\left\|u_{n}-y_{n}\right\|^{2}-c_{2}\left\|y_{n}-z_{n}\right\|^{2}\right] .
$$

Combining (3.8) and (3.9) provides that

$$
\begin{aligned}
\left\langle u_{n}-z_{n}, z_{n}-\wp^{*}\right\rangle \geqslant & \chi_{n+1}\left[\chi_{n}\left\{f\left(u_{n}, z_{n}\right)-f\left(u_{n}, y_{n}\right)\right\}\right. \\
& \left.-c_{1} \chi_{n}\left\|u_{n}-y_{n}\right\|^{2}-c_{2} \chi_{n}\left\|z_{n}-y_{n}\right\|^{2}\right]
\end{aligned}
$$

From (3.6) and (3.10), we obtain

$$
\begin{aligned}
2\left\langle u_{n}-z_{n}, z_{n}-\wp^{*}\right\rangle \geqslant & x_{n+1}\left[2\left\langle u_{n}-y_{n}, z_{n}-y_{n}\right\rangle\right. \\
& \left.-2 c_{1} \chi_{n}\left\|u_{n}-y_{n}\right\|^{2}-2 c_{2} x_{n}\left\|z_{n}-y_{n}\right\|^{2}\right] .
\end{aligned}
$$

We have the following facts:

$$
\begin{array}{r}
2\left\langle u_{n}-z_{n}, z_{n}-\wp^{*}\right\rangle=\left\|u_{n}-\wp^{*}\right\|^{2}-\left\|z_{n}-u_{n}\right\|^{2}-\left\|z_{n}-\wp^{*}\right\|^{2}, \\
2\left\langle u_{n}-y_{n}, z_{n}-y_{n}\right\rangle=\left\|u_{n}-y_{n}\right\|^{2}+\left\|z_{n}-y_{n}\right\|^{2}-\left\|u_{n}-z_{n}\right\|^{2} .
\end{array}
$$

The above relations with (3.11), provides the following

$$
\begin{aligned}
\left\|z_{n}-\wp^{*}\right\|^{2} \leqslant & \left\|u_{n}-\wp^{*}\right\|^{2}-\left(1-\chi_{n+1}\right)\left\|z_{n}-u_{n}\right\|^{2} \\
& -\chi_{n+1}\left(1-2 c_{1} \chi_{n}\right)\left\|u_{n}-y_{n}\right\|^{2}-\chi_{n+1}\left(1-2 c_{2} \chi_{n}\right)\left\|z_{n}-y_{n}\right\|^{2} .
\end{aligned}
$$


From (3.12), we obtain

$$
\left\|z_{n}-\wp^{*}\right\|^{2} \leqslant\left\|u_{n}-\wp^{*}\right\|^{2}, \forall n \geqslant 1 .
$$

It is given that $\wp^{*} \in S_{E P}$. From the definition of sequence $\left\{u_{n+1}\right\}$ and due to the fact that $g$ is a contraction with $\xi \in[0,1)$, we have

$$
\begin{aligned}
\left\|u_{n+1}-\wp^{*}\right\| & =\left\|\alpha_{n} g\left(u_{n}\right)+\left(1-\alpha_{n}\right) z_{n}-\wp^{*}\right\| \\
& =\left\|\alpha_{n}\left[g\left(u_{n}\right)-\wp^{*}\right]+\left(1-\alpha_{n}\right)\left[z_{n}-\wp^{*}\right]\right\| \\
& =\left\|\alpha_{n}\left[g\left(u_{n}\right)+g\left(\wp^{*}\right)-g\left(\wp^{*}\right)-\wp^{*}\right]+\left(1-\alpha_{n}\right)\left[z_{n}-\wp^{*}\right]\right\| \\
& \leqslant \alpha_{n}\left\|g\left(u_{n}\right)-g\left(\wp^{*}\right)\right\|+\alpha_{n}\left\|g\left(\wp^{*}\right)-\wp^{*}\right\|+\left(1-\alpha_{n}\right)\left\|z_{n}-\wp^{*}\right\| \\
& \leqslant \alpha_{n} \xi\left\|u_{n}-\wp^{*}\right\|+\alpha_{n}\left\|g\left(\wp^{*}\right)-\wp^{*}\right\|+\left(1-\alpha_{n}\right)\left\|z_{n}-\wp^{*}\right\| .
\end{aligned}
$$

Combining (3.13) and (3.14) and $\alpha_{n} \subset(0,1)$, this is what we deduce.

$$
\begin{aligned}
\left\|u_{n+1}-\wp^{*}\right\| & \leqslant \alpha_{n} \xi\left\|u_{n}-\wp^{*}\right\|+\alpha_{n}\left\|g\left(\wp^{*}\right)-\wp^{*}\right\|+\left(1-\alpha_{n}\right)\left\|u_{n}-\wp^{*}\right\| \\
& =\left[1-\alpha_{n}+\xi \alpha_{n}\right]\left\|u_{n}-\wp^{*}\right\|+\alpha_{n}(1-\xi) \frac{\left\|g\left(\wp^{*}\right)-\wp^{*}\right\|}{(1-\xi)} \\
& \leqslant \max \left\{\left\|u_{n}-\wp^{*}\right\|, \frac{\left\|g\left(\wp^{*}\right)-\wp^{*}\right\|}{(1-\xi)}\right\} \\
& \leqslant \max \left\{\left\|u_{0}-\wp^{*}\right\|, \frac{\left\|g\left(\wp^{*}\right)-\wp^{*}\right\|}{(1-\xi)}\right\} .
\end{aligned}
$$

Thus, we conclude that the $\left\{u_{n}\right\}$ is bounded sequence. This completes the proof of Lemma.

Theorem 3.2. Assume that $\left\{u_{n}\right\}$ is a sequence generated by Algorithm 1 and $\wp^{*} \in S_{E P} \neq \emptyset$. Then, $\left\{u_{n}\right\}$ converges strongly to $\wp^{*}=\mathrm{P}_{\mathrm{S}_{\mathrm{EP}}} \circ \mathrm{g}\left(\wp^{*}\right)$.

Proof. Now, we prove the strong convergence of the iterative sequence $\left\{u_{n}\right\}$ generated by Algorithm 1 . The Lipschitz-continuity and pseudo-monotone property of the bi-function $f$ implies that the solution set $S_{E P}$ is a closed and convex set (for more details see [31]). Since the mapping is a contraction and so does $P_{S_{E P}} \circ g$. Now, we are in position to use the Banach contraction theorem for the existence of a unique fixed point $\wp^{*} \in S_{E P}$ such that

$$
\wp^{*}=\mathrm{P}_{\mathrm{S}_{\mathrm{EP}}}\left(\mathrm{g}\left(\wp^{*}\right)\right)
$$

By using useful result of projection mapping, we have

$$
\left\langle\mathrm{g}\left(\wp^{*}\right)-\wp^{*}, y-\wp^{*}\right\rangle \leqslant 0, \forall y \in S_{\mathrm{EP}} .
$$

By Lemma 2.4 (i) and (3.12), we have

$$
\begin{aligned}
&\left\|u_{n+1}-\wp^{*}\right\|^{2} \\
&=\left\|\alpha_{n} g\left(u_{n}\right)+\left(1-\alpha_{n}\right) z_{n}-\wp^{*}\right\|^{2} \\
&=\left\|\alpha_{n}\left[g\left(u_{n}\right)-\wp^{*}\right]+\left(1-\alpha_{n}\right)\left[z_{n}-\wp^{*}\right]\right\|^{2} \\
&= \alpha_{n}\left\|g\left(u_{n}\right)-\wp^{*}\right\|^{2}+\left(1-\alpha_{n}\right)\left\|z_{n}-\wp^{*}\right\|^{2}-\alpha_{n}\left(1-\alpha_{n}\right)\left\|g\left(u_{n}\right)-z_{n}\right\|^{2} \\
& \leqslant \alpha_{n}\left\|g\left(u_{n}\right)-\wp^{*}\right\|^{2}+\left(1-\alpha_{n}\right)\left[\left\|u_{n}-\wp^{*}\right\|^{2}-\left(1-\chi_{n+1}\right)\left\|z_{n}-u_{n}\right\|^{2}\right. \\
&\left.-x_{n+1}\left(1-2 c_{1} \chi_{n}\right)\left\|u_{n}-y_{n}\right\|^{2}-\chi_{n+1}\left(1-2 c_{2} \chi_{n}\right)\left\|z_{n}-y_{n}\right\|^{2}\right]-\alpha_{n}\left(1-\alpha_{n}\right)\left\|g\left(u_{n}\right)-z_{n}\right\|^{2} \\
& \leqslant \alpha_{n}\left\|g\left(u_{n}\right)-\wp^{*}\right\|^{2}+\left\|u_{n}-\wp^{*}\right\|^{2} \\
&-\left(1-\alpha_{n}\right)\left[\left(1-\chi_{n+1}\right)\left\|z_{n}-u_{n}\right\|^{2}-x_{n+1}\left(1-2 c_{1} \chi_{n}\right)\left\|u_{n}-y_{n}\right\|^{2}-\chi_{n+1}\left(1-2 c_{2} x_{n}\right)\left\|z_{n}-y_{n}\right\|^{2}\right] .
\end{aligned}
$$


The above expression implies that

$$
\begin{aligned}
& \left(1-\alpha_{n}\right)\left[\left(1-\chi_{n+1}\right)\left\|z_{n}-u_{n}\right\|^{2}-\chi_{n+1}\left(1-2 c_{1} \chi_{n}\right)\left\|u_{n}-y_{n}\right\|^{2}-\chi_{n+1}\left(1-2 c_{2} \chi_{n}\right)\left\|z_{n}-y_{n}\right\|^{2}\right] \\
& \leqslant \alpha_{n}\left\|g\left(u_{n}\right)-\wp^{*}\right\|^{2}+\left\|u_{n}-\wp^{*}\right\|^{2}-\left\|u_{n+1}-\wp^{*}\right\|^{2} .
\end{aligned}
$$

The remainder of the proof shall be split into the following two parts:

Case 1: Assume that there is a fixed number $N_{1} \in \mathbb{N}\left(N_{1} \geqslant 1\right)$ such that

$$
\left\|u_{n+1}-\wp^{*}\right\| \leqslant\left\|u_{n}-\wp^{*}\right\|, \forall n \geqslant N_{1} .
$$

Then, $\lim _{n \rightarrow \infty}\left\|u_{n}-\wp^{*}\right\|$ exists. Let $\lim _{n \rightarrow \infty}\left\|u_{n}-\wp^{*}\right\|=$ l. By the use of (3.17), we obtain

$$
\begin{aligned}
& \left(1-\alpha_{n}\right)\left[\left(1-\chi_{n+1}\right)\left\|z_{n}-u_{n}\right\|^{2}-\chi_{n+1}\left(1-2 c_{1} \chi_{n}\right)\left\|u_{n}-y_{n}\right\|^{2}-\chi_{n+1}\left(1-2 c_{2} \chi_{n}\right)\left\|z_{n}-y_{n}\right\|^{2}\right] \\
& \leqslant \alpha_{n}\left\|g\left(u_{n}\right)-\wp^{*}\right\|^{2}+\left\|u_{n}-\wp^{*}\right\|^{2}-\left\|u_{n+1}-\wp^{*}\right\|^{2} .
\end{aligned}
$$

Thus, the $\lim _{n \rightarrow \infty}\left\|u_{n}-\wp^{*}\right\|$ exists and $\alpha_{n} \rightarrow 0$, we obtain

$$
\lim _{n \rightarrow \infty}\left\|z_{n}-u_{n}\right\|=\lim _{n \rightarrow \infty}\left\|u_{n}-y_{n}\right\|=\lim _{n \rightarrow \infty}\left\|z_{n}-y_{n}\right\|=0 .
$$

Furthermore, we have

$$
\begin{aligned}
\left\|u_{n+1}-u_{n}\right\| & =\left\|\alpha_{n} g\left(u_{n}\right)+\left(1-\alpha_{n}\right) z_{n}-u_{n}\right\| \\
& =\left\|\alpha_{n}\left[g\left(u_{n}\right)-u_{n}\right]+\left(1-\alpha_{n}\right)\left[z_{n}-u_{n}\right]\right\| \\
& \leqslant \alpha_{n}\left\|g\left(u_{n}\right)-u_{n}\right\|+\left(1-\alpha_{n}\right)\left\|z_{n}-u_{n}\right\| \rightarrow 0 .
\end{aligned}
$$

The term referred to above implies that

$$
\lim _{n \rightarrow \infty}\left\|u_{n+1}-u_{n}\right\|=0 .
$$

Thus, implies that the sequences $\left\{y_{n}\right\}$ and $\left\{z_{n}\right\}$ are bounded. Let $\left\{u_{n_{k}}\right\}$ be subsequence of $\left\{u_{n}\right\}$ such that $\left\{u_{n_{k}}\right\}$ converges weakly to $\hat{u} \in \mathcal{E}$. Next, we need to prove that $\hat{u} \in S_{E P}$. Due to the inequality (3.4), (3.6) and (3.9), we obtain

$$
\begin{aligned}
\mu \chi_{n_{k}} f\left(y_{n_{k}}, y\right) \geqslant & \mu \chi_{n_{k}} f\left(y_{n_{k}}, z_{n_{k}}\right)+\left\langle u_{n_{k}}-z_{n_{k}}, y-z_{n_{k}}\right\rangle \\
\geqslant & \chi_{n_{k}} \chi_{n_{k}+1} f\left(u_{n_{k}}, z_{n_{k}}\right)-\chi_{n_{k}} \chi_{n_{k}+1} f\left(u_{n_{k}}, y_{n_{k}}\right)-c_{1} \chi_{n_{k}} \chi_{n_{k}+1}\left\|u_{n_{k}}-y_{n_{k}}\right\|^{2} \\
& -c_{2} \chi_{n_{k}} \chi_{n_{k}+1}\left\|y_{n_{k}}-z_{n_{k}}\right\|^{2}+\left\langle u_{n_{k}}-z_{n_{k}}, y-z_{n_{k}}\right\rangle \\
\geqslant & \chi_{n_{k}+1}\left\langle u_{n_{k}}-y_{n_{k}}, z_{n_{k}}-y_{n_{k}}\right\rangle-c_{1} \chi_{n_{k}} \chi_{n_{k}+1}\left\|u_{n_{k}}-y_{n_{k}}\right\|^{2} \\
& -c_{2} \chi_{n_{k}} \chi_{n_{k}+1}\left\|y_{n_{k}}-z_{n_{k}}\right\|^{2}+\left\langle u_{n_{k}}-z_{n_{k}}, y-z_{n_{k}}\right\rangle
\end{aligned}
$$

where $y$ is an arbitrary member in $\varepsilon_{n}$. From (3.21), (3.23) and the boundedness of $\left\{u_{n}\right\}$ imply that righthand side goes to zero. From $\chi_{n_{k}}>0$, the condition (Ф3) and $y_{n_{k}} \rightarrow \hat{u}$, we obtain

$$
0 \leqslant \limsup _{k \rightarrow \infty} f\left(y_{n_{k}}, y\right) \leqslant f(\hat{u}, y), \forall y \in \mathcal{E}_{n} .
$$

It follows that $f(\hat{u}, y) \geqslant 0$, for all $y \in \mathcal{K}$, and hence $\hat{u} \in S_{E P}$. Next, we consider

$$
\begin{aligned}
& \limsup _{n \rightarrow \infty}\left\langle g\left(\wp^{*}\right)-\wp^{*}, u_{n}-\wp^{*}\right\rangle \\
& =\limsup _{k \rightarrow \infty}\left\langle g\left(\wp^{*}\right)-\wp^{*}, u_{n_{k}}-\wp^{*}\right\rangle=\left\langle g\left(\wp^{*}\right)-\wp^{*}, \hat{u}-\wp^{*}\right\rangle \leqslant 0 .
\end{aligned}
$$


Due to the use of $\lim _{n \rightarrow \infty}\left\|u_{n+1}-u_{n}\right\|=0$. We deduce that

$$
\begin{aligned}
& \limsup _{n \rightarrow \infty}\left\langle g\left(\wp^{*}\right)-\wp^{*}, u_{n+1}-\wp^{*}\right\rangle \\
& \leqslant \limsup _{n \rightarrow \infty}\left\langle g\left(\wp^{*}\right)-\wp^{*}, u_{n+1}-u_{n}\right\rangle+\limsup _{n \rightarrow \infty}\left\langle g\left(\wp^{*}\right)-\wp^{*}, u_{n}-\wp^{*}\right\rangle \leqslant 0 .
\end{aligned}
$$

By the use of Lemma 2.4(ii) and (3.13), we obtain

$$
\begin{aligned}
& \left\|u_{n+1}-\wp^{*}\right\|^{2} \\
& =\left\|\alpha_{n} g\left(u_{n}\right)+\left(1-\alpha_{n}\right) z_{n}-\wp^{*}\right\|^{2} \\
& =\left\|\alpha_{n}\left[g\left(u_{n}\right)-\wp^{*}\right]+\left(1-\alpha_{n}\right)\left[z_{n}-\wp^{*}\right]\right\|^{2} \\
& \leqslant\left(1-\alpha_{n}\right)^{2}\left\|z_{n}-\wp^{*}\right\|^{2}+2 \alpha_{n}\left\langle g\left(u_{n}\right)-\wp^{*},\left(1-\alpha_{n}\right)\left[z_{n}-\wp^{*}\right]+\alpha_{n}\left[g\left(u_{n}\right)-\wp^{*}\right]\right\rangle \\
& =\left(1-\alpha_{n}\right)^{2}\left\|z_{n}-\wp^{*}\right\|^{2}+2 \alpha_{n}\left\langle g\left(u_{n}\right)-g\left(\wp^{*}\right)+g\left(\wp^{*}\right)-\wp^{*}, u_{n+1}-\wp^{*}\right\rangle \\
& =\left(1-\alpha_{n}\right)^{2}\left\|z_{n}-\wp^{*}\right\|^{2}+2 \alpha_{n}\left\langle g\left(u_{n}\right)-g\left(\wp^{*}\right), u_{n+1}-\wp^{*}\right\rangle+2 \alpha_{n}\left\langle g\left(\wp^{*}\right)-\wp^{*}, u_{n+1}-\wp^{*}\right\rangle \\
& \leqslant\left(1-\alpha_{n}\right)^{2}\left\|z_{n}-\wp^{*}\right\|^{2}+2 \alpha_{n} \xi\left\|u_{n}-\wp^{*}\right\|\left\|u_{n+1}-\wp^{*}\right\|+2 \alpha_{n}\left\langle g\left(\wp^{*}\right)-\wp^{*}, u_{n+1}-\wp^{*}\right\rangle \\
& \leqslant\left(1+\alpha_{n}^{2}-2 \alpha_{n}\right)\left\|u_{n}-\wp^{*}\right\|^{2}+2 \alpha_{n} \xi\left\|u_{n}-\wp^{*}\right\|^{2}+2 \alpha_{n}\left\langle g\left(\wp^{*}\right)-\wp^{*}, u_{n+1}-\wp^{*}\right\rangle \\
& =\left(1-2 \alpha_{n}\right)\left\|u_{n}-\wp^{*}\right\|^{2}+\alpha_{n}^{2}\left\|u_{n}-\wp^{*}\right\|^{2}+2 \alpha_{n} \xi\left\|u_{n}-\wp^{*}\right\|^{2}+2 \alpha_{n}\left\langle g\left(\wp^{*}\right)-\wp^{*}, u_{n+1}-\wp^{*}\right\rangle \\
& =\left[1-2 \alpha_{n}(1-\xi)\right]\left\|u_{n}-\wp^{*}\right\|^{2}+2 \alpha_{n}(1-\xi)\left[\frac { \alpha _ { n } \| u _ { n } - \wp ^ { * } \| ^ { 2 } } { 2 ( 1 - \xi ) } \left\langle\frac{\left\langle g\left(\wp^{*}\right)-\wp^{*}, u_{n+1}-\wp^{*}\right\rangle}{1-\xi}\right.\right.
\end{aligned}
$$

It is clear from the expressions (3.27) and (3.28) that we obtain

$$
\limsup _{n \rightarrow \infty}\left[\frac{\alpha_{n}\left\|u_{n}-\wp^{*}\right\|^{2}}{2(1-\xi)}+\frac{\left\langle g\left(\wp^{*}\right)-\wp^{*}, u_{n+1}-\wp^{*}\right\rangle}{1-\xi}\right] \leqslant 0 .
$$

By taking $n \geqslant N_{2} \in \mathbb{N}\left(N_{2} \geqslant N_{1}\right)$ substantial sufficient such that $2 \alpha_{n}(1-\xi)<1$. By using (3.28), (3.29) and applying Lemma 2.2, we obtain that $\left\|u_{n}-\wp^{*}\right\| \rightarrow 0$ as $n \rightarrow \infty$.

Case 2: Assume there is a subsequence $\left\{n_{i}\right\}$ of $\{n\}$ such that

$$
\left\|u_{n_{i}}-\wp^{*}\right\| \leqslant\left\|u_{n_{i+1}}-\wp^{*}\right\|, \forall i \in \mathbb{N} .
$$

Thus, by Lemma 2.3 there exists a sequence $\left\{m_{k}\right\} \subset \mathbb{N}$ as $\left\{m_{k}\right\} \rightarrow \infty$, such that

$$
\left\|u_{m_{k}}-\wp^{*}\right\| \leqslant\left\|u_{m_{k+1}}-\wp^{*}\right\| \text { and }\left\|u_{k}-\wp^{*}\right\| \leqslant\left\|u_{m_{k+1}}-\wp^{*}\right\|, \text { for all } k \in \mathbb{N} \text {. }
$$

As the similar to Case 1, the expression (3.17) gives that

$$
\begin{gathered}
\left(1-\alpha_{m_{k}}\right)\left[\left(1-\chi_{m_{k}+1}\right)\left\|z_{m_{k}}-u_{m_{k}}\right\|^{2}-\chi_{m_{k}+1}\left(1-2 c_{1} \chi_{m_{k}}\right)\left\|u_{m_{k}}-y_{m_{k}}\right\|^{2}-\chi_{m_{k}+1}\left(1-2 c_{2} \chi_{m_{k}}\right)\left\|z_{m_{k}}-y_{m_{k}}\right\|^{2}\right] \\
\leqslant \alpha_{m_{k}}\left\|g\left(u_{m_{k}}\right)-\wp^{*}\right\|^{2}+\left\|u_{m_{k}}-\wp^{*}\right\|^{2}-\left\|u_{m_{k}+1}-\wp^{*}\right\|^{2} .
\end{gathered}
$$

By the use of $\alpha_{m_{k}} \rightarrow 0$, we get the following:

$$
\lim _{k \rightarrow \infty}\left\|z_{m_{k}}-u_{m_{k}}\right\|=\lim _{k \rightarrow \infty}\left\|u_{m_{k}}-y_{m_{k}}\right\|=\lim _{k \rightarrow \infty}\left\|z_{m_{k}}-y_{m_{k}}\right\|=0 .
$$

Next, we obtain

$$
\left\|u_{m_{k}+1}-u_{m_{k}}\right\|=\left\|\alpha_{m_{k}} g\left(u_{m_{k}}\right)+\left(1-\alpha_{m_{k}}\right) z_{m_{k}}-u_{m_{k}}\right\|
$$




$$
\begin{aligned}
& =\left\|\alpha_{m_{k}}\left[g\left(u_{m_{k}}\right)-u_{m_{k}}\right]+\left(1-\alpha_{m_{k}}\right)\left[z_{m_{k}}-u_{m_{k}}\right]\right\| \\
& \leqslant \alpha_{m_{k}}\left\|g\left(u_{m_{k}}\right)-u_{m_{k}}\right\|+\left(1-\alpha_{m_{k}}\right)\left\|z_{m_{k}}-u_{m_{k}}\right\| \rightarrow 0 .
\end{aligned}
$$

We must use the same argument as in Case 1 in order to do so

$$
\limsup _{k \rightarrow \infty}\left\langle g\left(\wp^{*}\right)-\wp^{*}, u_{m_{k}+1}-\wp^{*}\right\rangle \leqslant 0 .
$$

By using the expressions (3.28) and (3.30), we have

$$
\begin{aligned}
\left\|u_{m_{k}+1}-\wp^{*}\right\|^{2} \leqslant & {\left[1-2 \alpha_{m_{k}}(1-\xi)\right]\left\|u_{m_{k}}-\wp^{*}\right\|^{2} } \\
& +2 \alpha_{m_{k}}(1-\xi)\left[\frac{\alpha_{m_{k}}\left\|u_{m_{k}}-\wp^{*}\right\|^{2}}{2(1-\xi)}+\frac{\left\langle g\left(\wp^{*}\right)-\wp^{*}, u_{m_{k}+1}-\wp^{*}\right\rangle}{1-\xi}\right] \\
\leqslant & {\left[1-2 \alpha_{m_{k}}(1-\xi)\right]\left\|u_{m_{k}+1}-\wp^{*}\right\|^{2} } \\
& +2 \alpha_{m_{k}}(1-\xi)\left[\frac{\alpha_{m_{k}}\left\|u_{m_{k}}-\wp^{*}\right\|^{2}}{2(1-\xi)}+\frac{\left\langle g\left(\wp^{*}\right)-\wp^{*}, u_{m_{k}+1}-\wp^{*}\right\rangle}{1-\xi}\right] .
\end{aligned}
$$

It continues on from that

$$
\left\|u_{m_{k}+1}-\wp^{*}\right\|^{2} \leqslant \frac{\alpha_{m_{k}}\left\|u_{m_{k}}-\wp^{*}\right\|^{2}}{2(1-\xi)}+\frac{\left\langle g\left(\wp^{*}\right)-\wp^{*}, u_{m_{k}+1}-\wp^{*}\right\rangle}{1-\xi} .
$$

Since $\alpha_{m_{k}} \rightarrow 0$, and $\left\|u_{m_{k}}-\wp^{*}\right\|$ is a bounded sequence. Then, the expressions (3.34) and (3.36) indicate that

$$
\left\|u_{m_{k}+1}-\wp^{*}\right\|^{2} \rightarrow 0, \text { as } k \rightarrow \infty .
$$

The above implies that

$$
\lim _{k \rightarrow \infty}\left\|u_{k}-\wp^{*}\right\|^{2} \leqslant \lim _{k \rightarrow \infty}\left\|u_{m_{k}+1}-\wp^{*}\right\|^{2} \leqslant 0 .
$$

Consequently, $u_{n} \rightarrow \wp^{*}$. This completes the proof of the theorem.

\section{Applications}

Now, we consider the application of our main results to solve the problem of classic variational inequalities [30] for an operator $\mathcal{G}: \mathcal{E} \rightarrow \mathcal{E}$ is described as follows:

$$
\text { Fins } \wp^{*} \in \mathcal{K} \text { such that }\left\langle\mathcal{G}\left(\wp^{*}\right), y-\wp^{*}\right\rangle \geqslant 0, \forall y \in \mathcal{K} \text {. }
$$

We consider the following conditions to study variational inequalities.

(91) The solution set of the problem (VIP) denoted by $\mathrm{VI}(\mathcal{G}, \mathcal{K})$ is non-empty.

(G2) $\mathcal{G}: \mathcal{E} \rightarrow \mathcal{E}$ is called to be a pseudo-monotone, i.e.,

$$
\langle\mathcal{G}(u), y-u\rangle \geqslant 0 \Longrightarrow\langle\mathcal{G}(y), u-y\rangle \leqslant 0, \forall u, y \in \mathcal{K} \text {. }
$$

(93) $\mathcal{G}: \mathcal{E} \rightarrow \mathcal{E}$ is called to be a Lipschitz continuous if there exits a constants $L>0$ such that

$$
\|\mathcal{G}(\mathrm{u})-\mathcal{G}(\mathrm{y})\| \leqslant \mathrm{L}\|\mathrm{u}-\mathrm{y}\|, \forall \mathrm{u}, \mathrm{y} \in \mathcal{K} ;
$$

(G4) $\limsup _{n \rightarrow \infty}\left\langle\mathcal{G}\left(u_{n}\right), y-u_{n}\right\rangle \leqslant\left\langle\mathcal{G}\left(q^{*}\right), y-q^{*}\right\rangle$ for every $y \in \mathcal{C}$ and $\left\{u_{n}\right\} \subset \mathcal{C}$ satisfying $u_{n} \rightarrow q^{*}$. 
By the use of $f(u, y):=\langle\mathcal{G}(u), y-u\rangle$, for all $u, y \in \mathcal{K}$. Thus, our main problem turns into the problem of variational inequalities outlined above while $L=2 c_{1}=2 c_{2}$. From the above value of the bi-function $f$, we obtain

$$
\left\{\begin{array}{l}
y_{n}=\underset{y \in \mathcal{K}}{\arg \min }\left\{\chi_{n} f\left(u_{n}, y\right)+\frac{1}{2}\left\|u_{n}-y\right\|^{2}\right\}=P_{\mathcal{K}}\left(u_{n}-\chi_{n} \mathcal{G}\left(u_{n}\right)\right), \\
z_{n}=\underset{y \in \mathcal{E}_{n}}{\arg \min }\left\{\mu \chi_{n} f\left(y_{n}, y\right)+\frac{1}{2}\left\|u_{n}-y\right\|^{2}\right\}=P_{\mathcal{E}_{n}}\left(u_{n}-\mu \chi_{n} \mathcal{G}\left(y_{n}\right)\right) .
\end{array}\right.
$$

Corollary 4.1. Let $\mathcal{G}: \mathcal{K} \rightarrow \mathcal{E}$ be a mapping satisfying the conditions (G1)-(G4). Choose $\mathfrak{u}_{0} \in \mathcal{K}, 0<\sigma<$ $\min \left\{1, \frac{1}{\mathrm{~L}}\right\}, \mu \in(0, \sigma), \chi_{0}>0$ and a sequence $\alpha_{n} \subset(0,1)$ meet the following conditions, i.e.,

$$
\lim _{n \rightarrow \infty} \alpha_{n}=0 \text { and } \sum_{n=1}^{+\infty} \alpha_{n}=+\infty .
$$

Assume that $\left\{u_{n}\right\}$ generated as follows:

$$
\left\{\begin{array}{l}
y_{n}=P_{\mathcal{K}}\left(u_{n}-\chi_{n} \mathcal{G}\left(u_{n}\right)\right) \\
z_{n}=P_{\varepsilon_{n}}\left(u_{n}-\mu \chi_{n} \mathcal{G}\left(y_{n}\right)\right) \\
u_{n+1}=\alpha_{n} g\left(u_{n}\right)+\left(1-\alpha_{n}\right) z_{n}
\end{array}\right.
$$

where $\mathcal{E}_{n}=\left\{z \in \mathcal{E}:\left\langle u_{n}-\chi_{n} \mathcal{G}\left(u_{n}\right)-y_{n}, z-y_{n}\right\rangle \leqslant 0\right\}$. Update the step size rule in the following way:

$$
x_{n+1}=\left\{\begin{array}{c}
\min \left\{\sigma, \frac{\mu\left\langle\mathcal{G} y_{n}, z_{n}-y_{n}\right\rangle}{\left\langle\mathcal{G} u_{n}, z_{n}-y_{n}\right\rangle-\frac{L}{2}\left\|u_{n}-y_{n}\right\|^{2}-\frac{L}{2}\left\|z_{n}-y_{n}\right\|^{2}+1}\right\} \\
\quad \text { if } \frac{\mu\left\langle\mathcal{G} y_{n}, z_{n}-y_{n}\right\rangle}{\left\langle\mathcal{G} u_{n}, z_{n}-y_{n}\right\rangle-\frac{L}{2}\left\|u_{n}-y_{n}\right\|^{2}-\frac{L}{2}\left\|z_{n}-y_{n}\right\|^{2}+1}>0 \\
\chi_{0}
\end{array}\right.
$$

Then, the sequence $\left\{\mathrm{u}_{\mathrm{n}}\right\}$ converges strongly to $\wp^{*} \in \mathrm{VI}(\mathcal{G}, \mathcal{K})$.

Next, we study the application of our main results to figure out fixed-point problems associated with the $\mathrm{k}$-strict pseudo-contraction operator. A fixed point problem for an operator $\mathcal{T}: \mathcal{E} \rightarrow \mathcal{E}$ is defined as follows:

$$
\text { Find } \wp^{*} \in \mathcal{K} \text { such that } \mathcal{T}\left(\wp^{*}\right)=\wp^{*} \text {. }
$$

We assume that the following requirements have been met to study fixed point problems.

$(\mathfrak{T} 1) \mathcal{T}: \mathcal{K} \rightarrow \mathcal{K}$ is said to be a $\mathrm{k}$-strict pseudo-contraction [6] on $\mathcal{K}$ if

$$
\|\mathrm{Tu}-\mathrm{T} y\|^{2} \leqslant\|u-y\|^{2}+\kappa\|(u-T u)-(y-T y)\|^{2}, \forall u, y \in \mathcal{K} ;
$$

(T2) weakly sequentially continuous on $\mathcal{K}$ if

$$
\mathcal{T}\left(u_{n}\right) \rightarrow \mathcal{T}\left(\wp^{*}\right) \text { for any sequence in } \mathcal{K} \text { satisfy } u_{n} \rightarrow \wp^{*} .
$$

If we consider that the mapping $\mathcal{T}$ is a $k$-strict pseudocontraction and weakly continuous then $f(u, y)=$ $\langle u-\mathcal{T} u, y-u\rangle$ satisfies the conditions $(\Phi 1)-(\Phi 4)$ and $2 c_{1}=2 c_{2}=\frac{3-2 \kappa}{1-\kappa}$. Thus, we have

$$
\left\{\begin{array}{l}
y_{n}=\underset{y \in \mathcal{K}}{\arg \min }\left\{\chi_{n} f\left(u_{n}, y\right)+\frac{1}{2}\left\|u_{n}-y\right\|^{2}\right\}=P_{\mathcal{K}}\left[u_{n}-\chi_{n}\left(u_{n}-\mathcal{T}\left(u_{n}\right)\right)\right] \\
z_{n}=\underset{y \in \mathcal{E}_{n}}{\arg \min }\left\{\mu \chi_{n} f\left(y_{n}, y\right)+\frac{1}{2}\left\|u_{n}-y\right\|^{2}\right\}=P_{\mathcal{E}_{n}}\left[u_{n}-\mu \chi_{n}\left(y_{n}-\mathcal{T}\left(y_{n}\right)\right)\right] .
\end{array}\right.
$$


Corollary 4.2. Let $\mathcal{K}$ be a nonempty, convex and closed subset of a Hilbert space $\mathcal{E}$ and $\mathcal{T}: \mathcal{K} \rightarrow \mathcal{K}$ is a $\mathrm{k}$-strict pseudo-contraction and weakly continuous with solution set $\mathrm{Fix}(\mathcal{T}) \neq \emptyset$. Choose $\mathrm{u}_{0} \in \mathcal{K}, 0<\sigma<\min \left\{1, \frac{1-\mathrm{k}}{3-2 \mathrm{~K}}\right\}$, $\mu \in(0, \sigma), \chi_{0}>0$ and a sequence $\alpha_{n} \subset(0,1)$ meet the following conditions, i.e.,

$$
\lim _{n \rightarrow \infty} \alpha_{n}=0 \text { and } \sum_{n=1}^{+\infty} \alpha_{n}=+\infty .
$$

Assume that $\left\{u_{n}\right\}$ generated as follows:

$$
\left\{\begin{array}{l}
y_{n}=P_{\mathcal{K}}\left[u_{n}-\chi_{n}\left(u_{n}-\mathcal{T}\left(u_{n}\right)\right)\right] \\
z_{n}=P_{\mathcal{E}_{n}}\left[u_{n}-\mu \chi_{n}\left(y_{n}-\mathcal{T}\left(y_{n}\right)\right)\right] \\
u_{n+1}=\alpha_{n} g\left(u_{n}\right)+\left(1-\alpha_{n}\right) z_{n} .
\end{array}\right.
$$

where $\mathcal{E}_{n}=\left\{z \in \mathcal{E}:\left\langle\left(1-x_{n}\right) u_{n}+x_{n} \mathcal{T}\left(u_{n}\right)-y_{n}, z-y_{n}\right\rangle \leqslant 0\right\}$. Compute

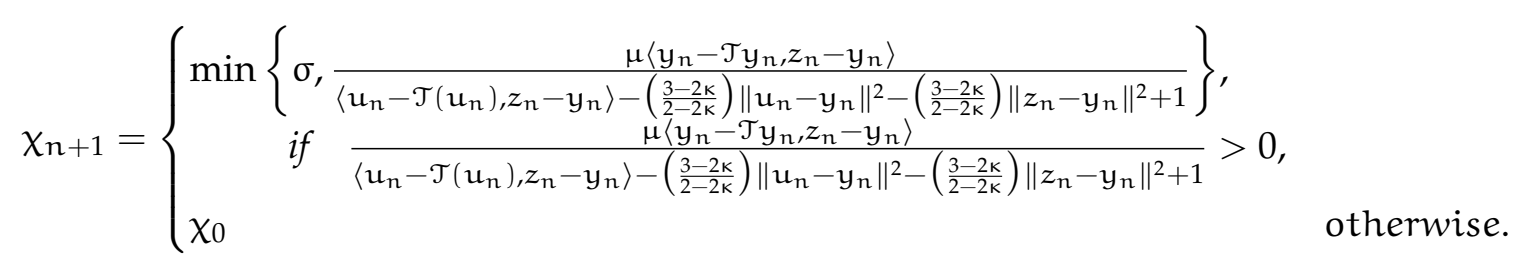

Then, sequence $\left\{u_{n}\right\}$ strongly converges to $\wp^{*} \in \operatorname{Fix}(\mathcal{T})$.

\section{Numerical Illustrations}

Numerical study results are provided in the following section to illustrate the effectiveness of the proposed methodology. To this end, we have studied one test problem in finite-dimensional spaces, and we have seen experiments on how the starting point affects the quality of the efficiency algorithms.

Example 5.1. Consider that $\mathrm{f}: \mathcal{K} \times \mathcal{K} \rightarrow \mathbb{R}$ is defined by

$$
f(u, y)=\langle P u+Q y+c, y-u\rangle, \forall u, y \in \mathcal{K},
$$

where $c \in \mathbb{R}^{n}$ and $\mathrm{P}, \mathrm{Q}$ are matrices of order $\mathrm{n}$. The matrix $\mathrm{P}$ is symmetric positive semi-definite and the matrix $\mathrm{Q}-\mathrm{P}$ is symmetric negative semi-definite with Lipschitz-type constants $\mathrm{c}_{1}=\mathrm{c}_{2}=\frac{1}{2}\|\mathrm{P}-\mathrm{Q}\|$ (see [31] for details). The matrices $\mathrm{P}, \mathrm{Q}$ and vector $\mathrm{c}$ are defined by

$$
\mathrm{P}=\left(\begin{array}{ccccc}
3.1 & 2 & 0 & 0 & 0 \\
2 & 3.6 & 0 & 0 & 0 \\
0 & 0 & 3.5 & 2 & 0 \\
0 & 0 & 2 & 3.3 & 0 \\
0 & 0 & 0 & 0 & 3
\end{array}\right) \quad \mathrm{Q}=\left(\begin{array}{ccccc}
1.6 & 1 & 0 & 0 & 0 \\
1 & 1.6 & 0 & 0 & 0 \\
0 & 0 & 1.5 & 1 & 0 \\
0 & 0 & 1 & 1.5 & 0 \\
0 & 0 & 0 & 0 & 2
\end{array}\right) \quad \mathrm{c}=\left(\begin{array}{c}
1 \\
-2 \\
-1 \\
2 \\
-1
\end{array}\right)
$$

The constraint set $\mathrm{C} \subset \mathbb{R}^{\mathrm{n}}$ is defined by

$$
C:=\left\{u \in \mathbb{R}^{n}:-10 \leqslant u_{i} \leqslant 10\right\} .
$$

The numerical and graphical results for three methods are shown in Figures 1-4 and Table 1 by using $u_{0}=y_{0}$. In addition, the control parameters conditions are taken in the following manner:

(i) $x=\frac{1}{4 c_{1}}, \alpha_{n}=\frac{1}{100(n+2)}$ and $D_{n}=\left\|u_{n+1}-u_{n}\right\|^{2}$ for Algorithm 2 (Hal-EgA) in [11]. 
(ii) $\chi_{n}=\frac{1}{7 c_{1}}, \alpha_{n}=\frac{1}{(n+1)^{0.5}}, D_{n}=\left\|u_{n+1}-u_{n}\right\|^{2}$ for Algorithm $3.1(\operatorname{Reg}-E g A)$ in [12] .

(iii) $\chi_{0}=0.15, \mu=0.33, \alpha_{n}=\frac{1}{100(n+2)}, g(u)=\frac{u}{3}$ and $D_{n}=\left\|u_{n+1}-u_{n}\right\|^{2}$ for Algorithm 1 (Vis-EgA).

Table 1: Numerical results values for Figures 1-5.

\begin{tabular}{cccc|ccc}
\hline \multicolumn{4}{c|}{ Number of iterations } & \multicolumn{3}{c}{ Elapsed time in seconds } \\
\hline $\mathbf{u}_{0}$ & Hal-EgA & Reg-EgA & Vis-EgA & Hal-EgA & Reg-EgA & Vis-EgA \\
\hline$(1,0,1,0,1)^{\top}$ & 19 & 24 & 11 & 0.169763 & 0.219508 & 0.103597 \\
$(1,2,-5,2,1)^{\top}$ & 26 & 31 & 17 & 0.267813 & 0.296752 & 0.167452 \\
$(0,1,-2,2,3)^{\mathrm{T}}$ & 24 & 30 & 16 & 0.219131 & 0.308820 & 0.167213 \\
$(3,-4,5,-4,3)^{\mathrm{T}}$ & 27 & 31 & 16 & 0.247530 & 0.282134 & 0.210123 \\
$(2,5,3,5,2)^{\mathrm{T}}$ & 23 & 27 & 14 & 0.210685 & 0.253114 & 0.179625 \\
\hline
\end{tabular}

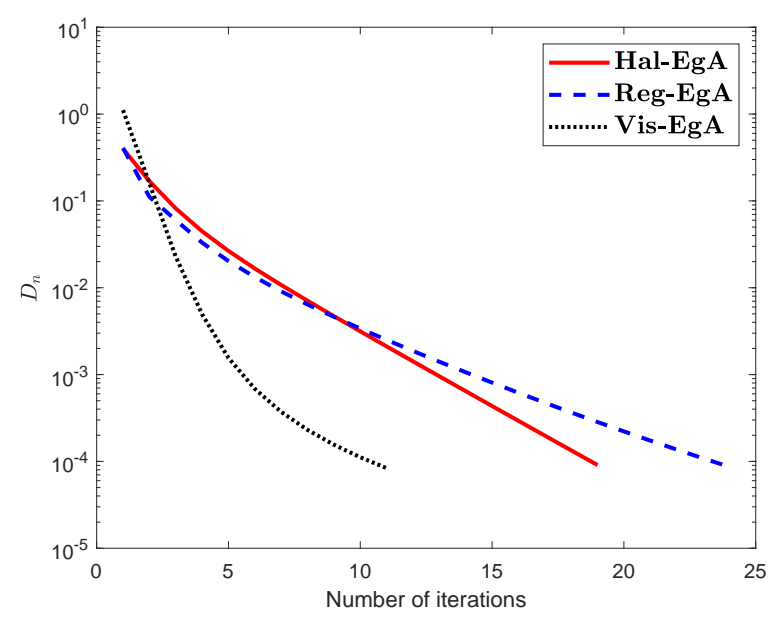

Figure 1: Numerical behaviour of Algorithm 1 with Algorithm 3.1 in [12] and Algorithm 2 in [11] when $\mathfrak{u}_{0}=(1,0,1,0,1)^{\top}$.

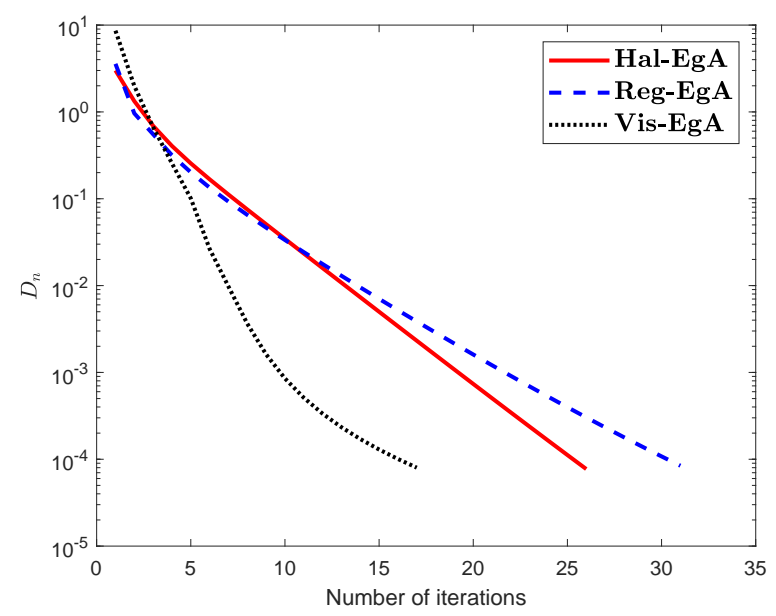

Figure 2: Numerical behaviour of Algorithm 1 with Algorithm 3.1 in [12] and Algorithm 2 in [11] when $\mathrm{u}_{0}=(1,2,-5,2,1)^{\top}$. 


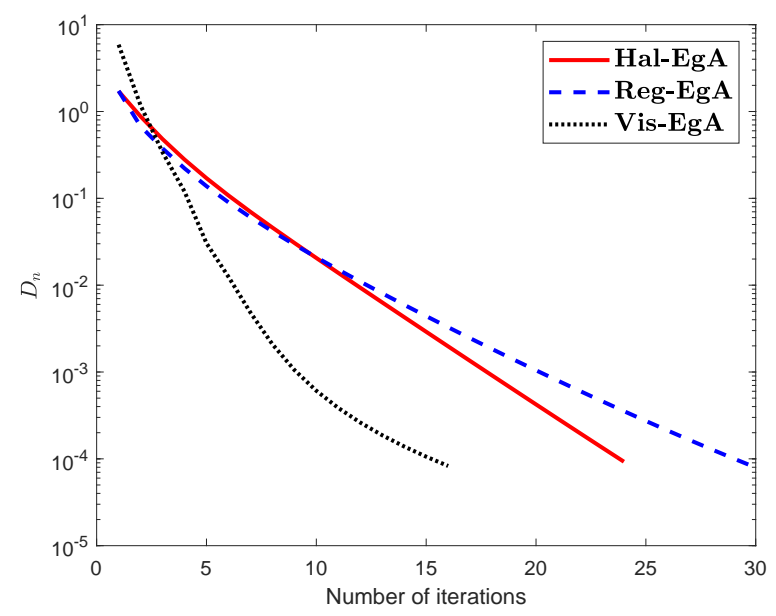

Figure 3: Numerical behaviour of Algorithm 1 with Algorithm 3.1 in [12] and Algorithm 2 in [11] when $u_{0}=(0,1,-2,2,3)^{\top}$.

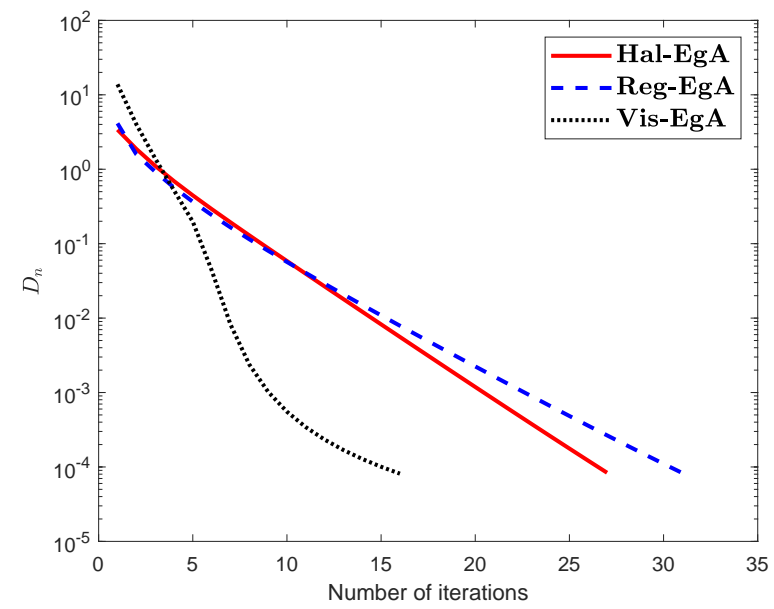

Figure 4: Numerical behaviour of Algorithm 1 with Algorithm 3.1 in [12] and Algorithm 2 in [11] when $u_{0}=(3,-4,5,-4,3)^{\top}$.

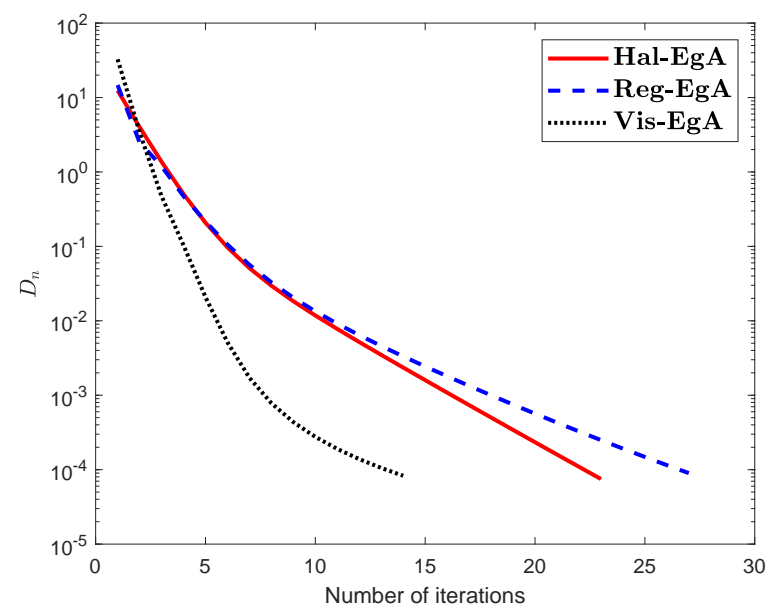

Figure 5: Numerical behaviour of Algorithm 1 with Algorithm 3.1 in [12] and Algorithm 2 in [11] when $\mathfrak{u}_{0}=(2,5,3,5,2)^{\top}$. 


\section{Conclusion}

We have designed a certain explicit viscosity type extragradient as a method to work out a pseudomonotone equilibrium problem in real Hilbert space, and we also verify that the layout sequence is strongly converging with the solution. The implementation of our results is being discussed in order to solve particular classes of equilibrium problems. Numerical conclusions have been drawn to explain the numerical efficiency of our algorithms compared to other methods. These numerical studies have shown that viscosity effects improve the efficiency of the iterative sequence in this context.

\section{Acknowledgment}

The authors are heartily grateful to the reviewers for their valuable remarks which greatly improved the results and presentation of the paper.

This project was supported by Rajamangala University of Technology Phra Nakhon (RMUTP).

\section{References}

[1] A. Antipin, Equilibrium programming: proximal methods, Comput. Math. Math. Phys., 37 (1997), 1285-1296. 1

[2] H. H. Bauschke, P. L. Combettes, Convex Analysis and Monotone Operator Theory in Hilbert Spaces, Springer, Cham, (2017). 2.1, 2.4

[3] M. Bianchi, S. Schaible, Generalized monotone bifunctions and equilibrium problems, J. Optim. Theory Appl., 90 (1996), 31-43. 2

[4] G. Bigi, M. Castellani, M. Pappalardo, M. Passacantando, Existence and solution methods for equilibria, Eur. J. Oper. Res., 227 (2013), 1-11. 1

[5] E. Blum, W. Oettli, From optimization and variational inequalities to equilibrium problems, Math. Student, 63 (1994), 123-145. 1, 1, 2

[6] F. E. Browder, W. V. Petryshyn, Construction of fixed points of nonlinear mappings in Hilbert space, J. Math. Anal. Appl., 20 (1967), 197-228. 4

[7] Y. Censor, A. Gibali, S. Reich, The subgradient extragradient method for solving variational inequalities in Hilbert space, J. Optim. Theory Appl., 148 (2011), 318-335. 1

[8] K. Fan, A minimax inequality and applications, Inequalities III, Academic Press, New York, (1972). 1

[9] S. D. Flåm, A. S. Antipin, Equilibrium programming using proximal-like algorithms, Math. Programming, 78 (1996), 29-41. 1

[10] F. Giannessi, A. Maugeri, P. M. Pardalos, Equilibrium problems: nonsmooth optimization and variational inequality models, Springer Science \& Business Media, New York, (2006). 1

[11] D. V. Hieu, Halpern subgradient extragradient method extended to equilibrium problems, Rev. R. Acad. Cienc. Exactas Fís. Nat. Ser. A Mat. RACSAM, 111 (2017), 823-840. 1, 5.1, 1, 2, 3, 4, 5

[12] D. V. Hieu, J. J. Strodiot, L. D. Muu, Strongly convergent algorithms by using new adaptive regularization parameter for equilibrium problems, J. Comput. Appl. Math., 376 (2020), 21 pages. 1, 5.1, 1, 2, 3, 4,5

[13] S. I. Lyashko, V. V. Semenov, A new two-step proximal algorithm of solving the problem of equilibrium programming, in Optimization and Its Applications in Control and Data Sciences, Springer, Cham, (2016). 1

[14] P.-E. Maingé, Strong convergence of projected subgradient methods for nonsmooth and nonstrictly convex minimization, Set-Valued Anal., 16 (2008), 899-912. 2.3

[15] G. Mastroeni, On auxiliary principle for equilibrium problems, in Nonconvex Optimization and Its Applications, Springer, Boston, (2003). 2

[16] A. Moudafi, Viscosity approximation methods for fixed-points problems, J. Math. Anal. Appl., 241 (2000), 46-55. 1, 3

[17] L. D. Muu, W. Oettli, Convergence of an adaptive penalty scheme for finding constrained equilibria, Nonlinear Anal., 18 (1992), 1159-1166. 1

[18] H. U. Rehman, P. Kumam, A. B. Abubakar, Y. J. Cho, The extragradient algorithm with inertial effects extended to equilibrium problems, Comput. Appl. Math., 39 (2020), 1-26. 1

[19] H. U. Rehman, P. Kumam, I. K. Argyros, N. A. Alreshidi, W. Kumam, W. Jirakitpuwapat, A self-adaptive extragradient methods for a family of pseudomonotone equilibrium programming with application in different classes of variational inequality problems, Symmetry, 12 (2020), 1-27. 1

[20] H. U. Rehman, P. Kumam, I. K. Argyros, W. Deebani, W. Kumam, Inertial extra-gradient method for solving a family of strongly pseudomonotone equilibrium problems in real Hilbert spaces with application in variational inequality problem, Symmetry, 12 (2020), 1-24.

[21] H. U. Rehman, P. Kumam, I. K. Argyros, M. Shutaywi, Z. Shah, Optimization based methods for solving the equilibrium problems with applications in variational inequality problems and solution of nash equilibrium models, Math., 8 (2020), 128. 1 
[22] H. U. Rehman, P. Kumam, Y. J. Cho, Y. I. Suleiman, W. Kumam, Modified Popov's explicit iterative algorithms for solving pseudomonotone equilibrium problems, Optim. Methods Softw., 36 (2021), 82-113. 1

[23] H. U. Rehman, P. Kumam, Y. J. Cho, P. Yordsorn, Weak convergence of explicit extragradient algorithms for solving equilibrium problems, J. Inequal. Appl., 2019 (2019), 1-25. 1

[24] H. U. Rehman, P. Kumam, Q.-L. Dong, Y. J. Cho, A modified self-adaptive extragradient method for pseudomonotone equilibrium problem in a real Hilbert space with applications, Math. Methods Appl. Sci., 44 (2021), 3527-3547. 1

[25] H. U. Rehman, P. Kumam, Q.-L. Dong, Y. Peng, W. Deebani, A new popov's subgradient extragradient method for two classes of equilibrium programming in a real Hilbert space, Optimization, (2020), 1-36. 1

[26] H. U. Rehman, P. Kumam, W. Kumam, M. Shutaywi, W. Jirakitpuwapat, The inertial sub-gradient extra-gradient method for a class of pseudo-monotone equilibrium problems, Symmetry, 12 (2020), 1-25. 1

[27] H. U. Rehman, P. Kumam, M. Shutaywi, N. A. Alreshidi, W. Kumam, Inertial optimization based two-step methods for solving equilibrium problems with applications in variational inequality problems and growth control equilibrium models, Energies, 13 (2020), 1-28. 1

[28] H. U. Rehman, N. Pakkaranang, P. Kumam, Y. J. Cho, Modified subgradient extragradient method for a family of pseudomonotone equilibrium problems in real a Hilbert space, J. Nonlinear Convex Anal., 21 (2020), 2011-2025. 1

[29] R. T. Rockafellar, Convex analysis, Princeton University Press, (1970). 2.5

[30] G. Stampacchia, Formes bilinéaires coercitives sur les ensembles convexes, C. R. Acad. Sci. Paris, 258 (1964), $4413-4416$. 4

[31] D. Q. Tran, M. L. Dung, V. H. Nguyen, Extragradient algorithms extended to equilibrium problems, Optimization, 57 (2008), 749-776. 1, 3, 3, 5.1

[32] H.-K. Xu, Another control condition in an iterative method for nonexpansive mappings, Bull. Aust. Math. Soc., 65 (2002), 109-113. 2.2

[33] P. Yordsorn, P. Kumam, H. U. Rehman, Modified two-step extragradient method for solving the pseudomonotone equilibrium programming in a real Hilbert space, Carpathian J. Math., 36 (2020), 313-330. 1 